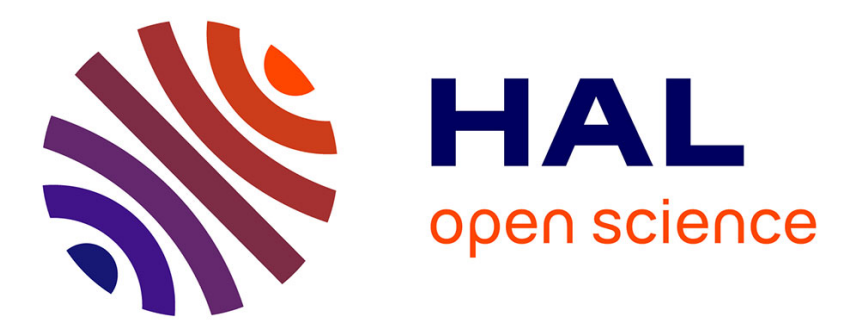

\title{
Effects of self-selected music and the arousal level of music on user experience and performance in video games
}

\author{
Arthur Abia, Loïc Caroux
}

\section{- To cite this version:}

Arthur Abia, Loïc Caroux. Effects of self-selected music and the arousal level of music on user experience and performance in video games. 20th Congress of the International Ergonomics Association IEA 2018, Aug 2018, Florence, Italy. pp.3-12, 10.1007/978-3-319-96059-3_1 . hal-01862435

\section{HAL Id: hal-01862435 \\ https://hal.science/hal-01862435}

Submitted on 27 Aug 2018

HAL is a multi-disciplinary open access archive for the deposit and dissemination of scientific research documents, whether they are published or not. The documents may come from teaching and research institutions in France or abroad, or from public or private research centers.
L'archive ouverte pluridisciplinaire HAL, est destinée au dépôt et à la diffusion de documents scientifiques de niveau recherche, publiés ou non, émanant des établissements d'enseignement et de recherche français ou étrangers, des laboratoires publics ou privés. 


\title{
Effects of Self-Selected Music and the Arousal Level of Music on User Experience and Performance in Video Games
}

\author{
Arthur Abia and Loïc Caroux $\bowtie$ [0000-0002-3794-9653] \\ CLLE (University of Toulouse \& CNRS), 5 allées Antonio Machado, 31058 Toulouse, France \\ arthurabia@gmail.com ; loic.caroux@univ-tlse2.fr
}

\begin{abstract}
Music is important in video games. It has to be considered more than just an environmental stimulus. However, the role of music in user experience in video games has received little attention in the literature. In particular, there is still a need for investigation of the effects of self-selected music and the arousal level of music on player experience. An experiment was designed to study the effects of these music characteristics on player performance and player experience in different genres of games. Although no significant effect on performance was observed, results showed that the type of music displayed when playing has an impact on players' affective enjoyment and music perception. Moreover, further analyses revealed significant interactions between the genre of game and the type of music presented on player experience. Overall, these findings suggest that music should be better taken in account in video game design to optimize player experience.
\end{abstract}

Keywords: Game design, Player experience, Enjoyment, Musical preference, Game genre.

\section{Introduction}

Music has become an important part of everyday life. A growing number of studies has been therefore dedicated to it [1]. In the field of cognitive ergonomics, music study is mainly task-performance oriented. According to the literature [2, 3], music can be considered as an additional distraction, interfering with the completion of a task. The concept of distraction, refers here to the process of attention, as defined by Broadbent [4]. In this model, attention is described as a cognitive filter, selecting and processing the most relevant information as well as inhibiting the other messages in goal-oriented tasks. According to this model, attention is a limited process. Processing stimuli during task execution or in the presence of external stimulus drains from the cognitive system of an operator or a user. Exposition to musical stimuli may then be detrimental performance-wise.

However, in video games, soundtracks are a common part of the player experience. They have to be considered more than ambient stimuli and they are not used just as auditory feedback anymore [5]. The role of music in user experience in video games 
has received little attention in the literature. There is still much to discover about the impact of audio in video games. In particular, there is still a need for investigation of the effects of self-selected music and the arousal level of music on player experience.

The present study aimed to investigate the effects of music, and more specifically the effects of musical preference and the arousal level of music on user performance and user experience in a given task: playing video games. Based on the work of Cassidy and MacDonald [6], an experiment was designed to assess the effects of these music characteristics on player performance and player experience in different types of games.

\subsection{Influence of Music on Human Behavior}

Musical preference has been widely studied in the literature of psychology of art. According to Berlyne [7], art can be expressed in terms of "aesthetics stimuli". The author suggested a theory in which the subjective experience of art, visually or auditory, may provoke a variation in arousal levels, both physiologically and psychologically. This variation of stimulation would be related to the characteristics of the experienced stimuli, such as their novelty, their complexity, or their tempo for example in the case of music. The preference one feels for any form of aesthetics stimulus would then be linked to the perceived degree of arousal.

Listening to music requires cognitive resources, music being a stimulus needing to be analyzed and processed. Konecni [8] studied the relation between musical preference and the listener's performance in a dual-task context. The author showed that the musical preference is influenced by the arousal provoked by music and also by the amount of cognitive resources available during the dual-task process. The more demanding a task is, the more intrusive a music is perceived, and the less it is appreciated.

The notion of musical preference is as well related to task performance. In a study conducted by Nantais and Schellenberg [9], the participants performed significantly better a given task when they listened to a stimulus they like, may it had been Schubert or even a story narrated by Stephen King.

\subsection{Influence of Music in Video Games}

Further literature investigated the influence of music in human-computer interaction situations, and in particular in player-video game interaction. With the advances in technology, more and more studies have been published on video games and user experience. While most of them examine the visual aspect of the game interface (see [10] for a review), a number of studies analyzed the auditory dimension of interactive games. For example, Nacke, Grimshaw and Lindley [5] have shown that the presence of sounds and/or music during gameplay was correlated to an increase of positive affects regarding subjective game experience and a decrease of negative ones.

Several studies focused on the topic of musical preference. A study conducted by North and Hargreaves [11] aimed to investigate the relation between music listening, musical preferences and performance in a concurrent task in a context of dual-task while playing a driving game. The authors found that the driving performance was lower when the participants had to listen to the high-arousal music while performing 
the concurrent task, due to a high cognitive workload. They also found that the driving performance was higher when the participants listened to the low-arousal music with no concurrent task. Finally, the authors reported higher ratings of music appreciation when there was no concurrent task to simultaneously perform. In line with Konecni [8], those results showed that musical preference in the context of task performance is related to the nature of the task, and to the cognitive resources available when listening.

More recent evidence of this phenomenon can be found in a study conducted by Cassidy and MacDonald [6]. The authors asked participants to perform a driving task in a video game. They were separated in 5 groups to compare several auditory conditions: no sound, car sounds alone, car sounds with the addition of self-selected music, car sounds with high-arousal music, and car sounds with low-arousal music. The authors found that the participants who had the opportunity to bring their own songs reported a higher level of appropriateness, lower levels of tension-anxiety, had a higher driving performance, and overall had a better user experience. They also found that the highest driving performance was found in the high-arousal music condition.

These studies showed that music is more than an additional information existing in the auditory dimension. It can be used as a tool to change levels of arousal as well as affects, thus having an effect in goal-oriented contexts. Depending on the task, one could allocate their preferences depending on the cognitive resources available. In the present study, those effects of music were investigated on a different type of material, to appreciate the external validity of previous studies.

\subsection{The Present Study}

The aim of the present study was to extend current knowledge of the effects of musical preference and arousal level of music on user experience and performance in video games. This investigation, in line with Konecni [8], aimed to measure the user experience and performance as well as taking into account the ecological choice of music self-selection according to the task to perform. Our experimental set up was based on the Cassidy and Macdonald's one [6]. The main differences are that, in the present study, enjoyment of players was measured with a specific questionnaire [12], and that two different types of games were used, namely an action game and a puzzle game. Most of studies tended to focus on driving games [11, 13]. With this in mind, our research tried to examine others contexts of video games to determine the external validity of past studies. The performance of players was measured in two distinct video games, as well as their subjective experience, several times under varying musical conditions for each session. There were three types of musical conditions: high-arousal music, low-arousal music and self-selected music. In this last condition, the music track was brought by the players. The user experience was measured using several existing questionnaires.

The following hypotheses were established on the basis of the previous literature described above. The first hypothesis was that the performance is overall better when participants are exposed to the music they select themselves in comparison to the other music conditions, for both types of games. The second hypothesis was that the subjective experience of players is better when exposed to self-selected music as compared to 
experimenter-selected music, for both types of games. The third hypothesis was selfselected music is more appreciated and perceived as more appropriate than other music conditions, for both types of games. The fourth hypothesis was that the player performance is better when high-arousal music is displayed during the action game than when low-arousal music is displayed, and vice-versa in the puzzle game. In a similar way, the fifth hypothesis was that high-arousal music is perceived as more appropriate in the action game than low-arousal music, and vice-versa in the puzzle game.

\section{Methods}

\subsection{Participants}

A total of 20 participants (10 women) from age 20 to $34(M=23.9, S D=4.1)$ were recruited for the study. They were university students from various disciplines, recruited on a voluntary basis, with various gaming expertise.

\subsection{Material}

Music. The music tracks used in for the low-arousal and high-arousal conditions were selected from the study of Cassidy and MacDonald [2] and prepared in advance. This choice allowed us to use an already empirically validated material. The different musical pieces used were "Distractions" from the album Simple Things (2002) of the band Zero 7 and "Attitude" from the album Roots (1996) of the band Sepultura. "Distractions" is a down-tempo music with a rhythm of 102 BPM and "Attitude" is a groove metal song with a rhythm of 155 BPM. Regarding the self-selected music, the participants were asked to choose a music before coming to the experimentation. They were asked to "choose a song they like, and that they would like to hear during the game session." Participants knew that the experiment was to play video games under several conditions, but they did not know the type of game in advance. Music was diffused with computer speakers.

Games. The selected video games were World of Goo (2D Boy, 2008) and Geometry Wars 3: Dimensions (Lucid Games, 2014). They were fairly recent and critically wellreceived. They were chosen for their typical genre-characteristics. World of Goo is a puzzle video game, where the goal is to assemble small creatures called "Goos" into structures, in order to lead them through an exit pipe. This game has a static environment, meaning that the situation on screen only moves when the players enter an input. Geometry Wars 3: Dimensions is an action video game, in which the player controls a spaceship and must earn the highest score possible by destroying enemies' spaceships. This game has a dynamic environment, meaning that the situation evolves independently from the players' action, and they have to adapt and react to it. For the action game, the instruction was to "destroy as many ships as possible while dying the least possible during 3 minutes" and for the puzzle game, it was to "finish the level as fast as possible using as few moves as possible". For this experimentation, only the music 
was manipulated. The original game sounds (coming from environment, enemies, effects, etc.) were not manipulated. They were always presented during the game sessions.

Questionnaire. The questionnaire was composed of items from the Fang et al.'s "instrument to measure enjoyment of computer game play" [12], and from the study of Cassidy and MacDonald [6], which assesses the players' perception of music in a video game and their global play experience. The Fang et al.'s [12] instrument aims to assess the level of enjoyment, by focusing on 3 dimensions affecting the user experience during gameplay: the affective aspect (related to emotions and feelings), the behavioral aspect (related to the behavior during the game session) and the cognitive aspect (related to the judgment of game elements by the player). We present only the affective dimension in the present paper. The questionnaire included 11 items (Table 1), which had to be rated on a 10-point Likert scale.

Table 1. Items used for the questionnaire.

\begin{tabular}{lll}
\hline Study of origin & Dimension & Item \\
\hline \multirow{3}{*}{ Fang et al. [12] } & $\begin{array}{l}\text { Affective aspect } \\
\text { of enjoyment }\end{array}$ & $\begin{array}{l}\text { 1. I feel unhappy when playing this game } \\
\text { 2. I feel worried when playing this game } \\
\text { 3. I feel happy when playing this game } \\
\text { 4. I feel exhausted when playing this game } \\
\text { 5. I feel miserable when playing this game }\end{array}$ \\
\hline \multirow{2}{*}{ Cassidy and } & Global experience & $\begin{array}{l}\text { 6. I felt distracted while playing } \\
\text { 7. I enjoyed the game experience }\end{array}$ \\
\cline { 2 - 3 } MacDonald [6] & \multirow{2}{*}{ Music perception } & $\begin{array}{l}\text { 8. I liked the music during playing } \\
\text { 9. I felt the music was familiar } \\
\text { 10. I felt the music was appropriate for the task } \\
\text { 11. I found the music arousing }\end{array}$ \\
\hline
\end{tabular}

\subsection{Design and Procedure}

The type of music (low-arousal vs. high-arousal vs. self-selected) and the genre of game (action vs. puzzle) were manipulated as within-participants factors. Once recruited, participants did not know which type of video game they were going to play to. Depending on the sessions' order, the respective video game was presented to the participant, as well as the instructions. The order of the game sessions was counter-balanced. Controls, using keyboard and mouse only, were explained before proceeding. After a training session without any music presented, the experiment began. Each participant completed 6 game sessions. The experiment was segmented in two blocks of 3 sessions for each game, each session corresponding to a different music condition. The order of music conditions was also counter-balanced for each participant. After each session, participants had to fill the subjective experience questionnaires. After 3 sessions with the first game presented, participants were then presented the second game of the experiment, 
with the same procedure. The mean time of a game session was 2 minutes, without the time of completing the questionnaire.

\subsection{Dependent Variables}

Performance indicators used for the action game were the score and the number of destroyed enemies for each session. The elapsed time as well as the number of moves done for each session were used for the puzzle game. The subjective experience of the participants was assessed with the answers given for each item of the questionnaire.

\section{$3 \quad$ Results}

\subsection{Performance}

The performance indicators were analyzed separately for each game because they were not similar and thus not readily comparable. A repeated measures ANOVA was carried out to investigate the differences between the music conditions on defined performance indicators. The ANOVA carried out did not present any significant difference for the score nor for the number of deaths in the action game, and neither for the elapsed time nor for the number of moves in the puzzle game.

\subsection{Questionnaire}

Items of the questionnaires were analyzed with the two independent variables, namely the type of music and the type of game, as well as their interaction. Repeated measures ANOVAs were performed for all items of the questionnaires.

Affective Aspect of Enjoyment. For Item 3 "I feel happy when playing this game", the main effect of the type of music $F(2,38)=6.10, p<.01, \eta^{2}{ }_{p}=.24$ on the answers of participants, as well as the interaction between the type of music and the type of game, $F(2,38)=5.08, p<.01, \eta^{2} p=.21$, were significant. As shown in Figure 1, participants reported more positive affect when exposed to self-selected music compared to other types of music for both types of game. It was also observed that in the action game, participants reported more positive affect with the high-arousal music than the lowarousal music, and vice-versa for the puzzle game.

For Item 2 "I feel worried when playing this game", there was a significant difference of the type of game $F(1,19)=4.96, p<.05, \eta^{2}=.21$ on the answers of participants, in the sense that the participants reported higher scores when playing the action game than the puzzle game. However, there were no significant difference between the types of music $F(2,38)=1.09, p=.35$. The interaction between these two factors did not reach significance $F(2,38)<1, p=.85$.

For the other items (1, 4 and 5), none of the observed differences were statistically significant neither for the type of game, nor for the type of music, or their interactions. 


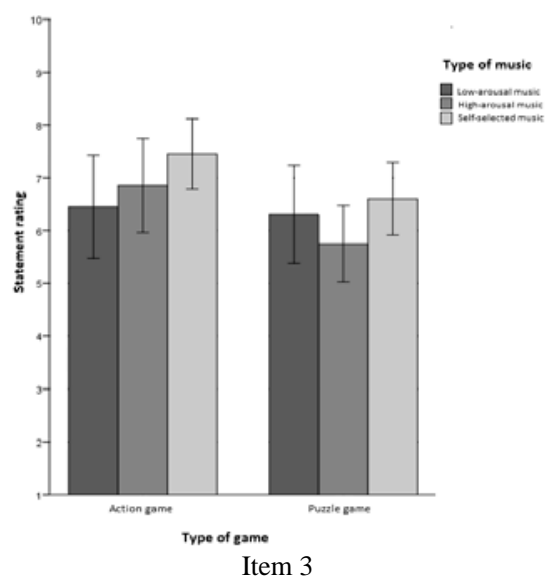

"I feel happy when playing this game"

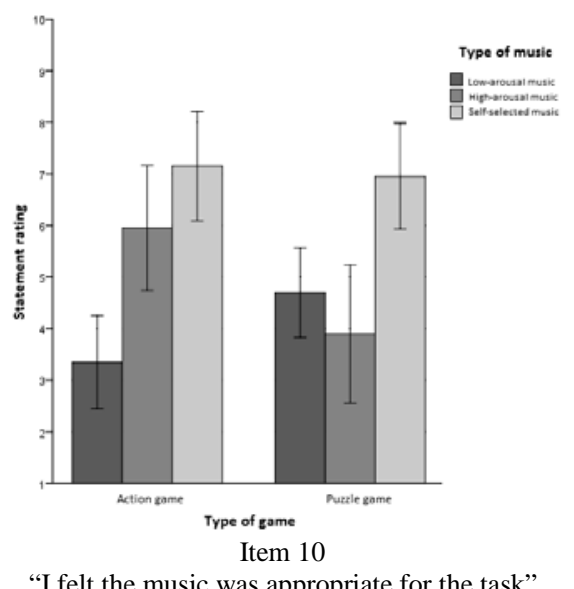

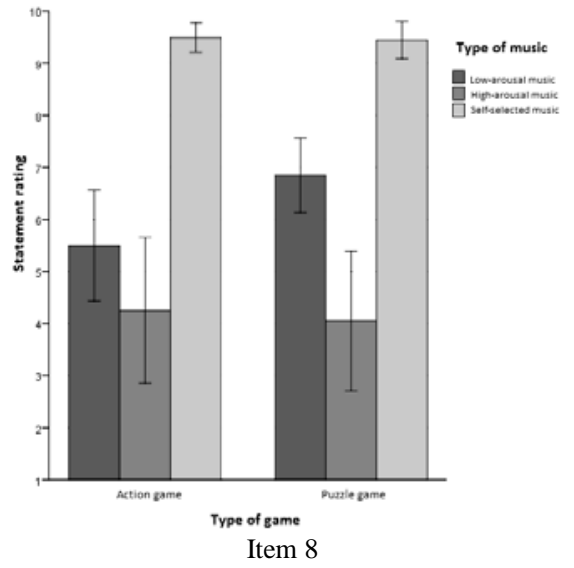

"I liked the music during playing"

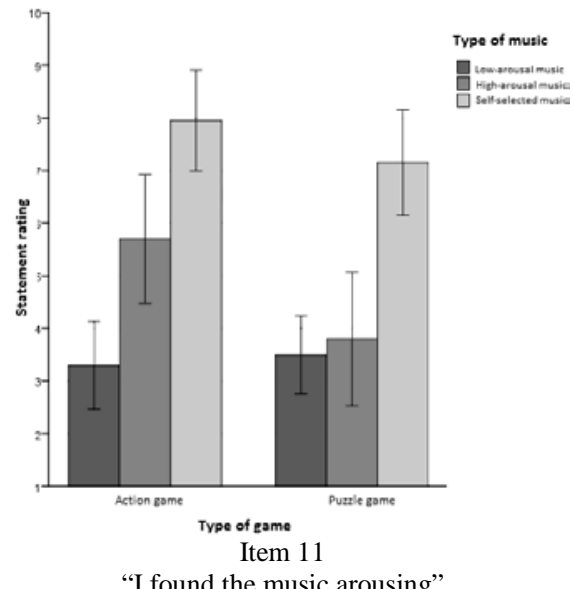

Fig. 1. Mean ratings for the items 3, 8, 10 and 11 for each type of game and type of music. The error bars represent standard errors.

Music Perception. For Item 8 "I liked the music during playing", there was a significant difference between the types of music, $F(2,38)=50.60, p<.001, \eta_{p}^{2}=.73$, and between the types of game, $F(1,19)=4.43, p<.05, \eta^{2} p=.19$. A significant interaction was observed between the type of music and the type of game $F(2,38)=4.87, p<.05$, $\eta_{p}^{2}=.20$. As shown in Figure 1, participants appreciated the most their self-selected music for both types of game. It was also observed that in the action game, participants appreciated more the low-arousal music in the puzzle game than in the action game.

For Item 10, "I felt the music was appropriate for the task", there was a significant difference between the two types of music $F(2,38)=15.52, p<.001, \eta^{2} p=.45$ as well as an interaction between the type of music and the type of game $F(2,38)=9.54, p<$ 
$.001, \eta_{p}^{2}=.33$. Figure 1 shows that self-selected music was perceived as more appropriate than the other types of music, independently from the type of game. Moreover, high-arousal music was perceived as more appropriate in the action game than in the puzzle game, and vice-versa for the low-arousal music. The analysis did not reveal any significant differences between the types of games $F(1,19)=.87, p=.36$.

The results for Item 11 "I found the music arousing” revealed a significant difference between the type of music $F(2,38)=39.32, p<.001, \eta^{2}=.67$ and the type of game $F(1,19)=8.16, p<.01, \eta^{2} p=.30$. There was also a significant interaction between the two variables $F(2,38)=3.57, p<.05, \eta_{p}^{2}=.16$. As shown in Figure 1 , self-selected music was perceived as more arousing than the other types of music for both types of game. Moreover, there was a significant difference of perceived arousal between higharousal and low-arousal music in the action game, but none in the puzzle game.

For Item 9 "I felt the music was familiar", there was a significant difference between the type of music $F(2,38)=70.54, p<.001, \eta_{p}^{2}=.79$, in the sense that participants reported higher scores for the self-selected music than the other music conditions, but none for the type of game $F(1,19)=1.49, p=.24$ or for the interaction of the two variables $F(2,38)=1.14, p=.33$.

Global Experience. Item 6 "I felt distracted while playing” showed a significant difference between the types of games $F(1,19)=10.70, p<.01, \eta^{2}{ }^{2}=.36$, in the sense that players reported higher scores when playing the puzzle game than the action game, but none for the type of music $F(2,38)=2.10, p=.14$ or for the interaction of the two variables $F(2,38)=2.32, p=.112$.

Item 7 "I enjoyed the game experience" also showed a significant difference between the types of games $F(1,19)=4.84, p<.05, \eta_{p}^{2}=.20$, in the sense that players reported higher scores when playing the action game than the puzzle game, but none for the type of music $F(2,38)=2.20, p=.13$ or for the interaction of the two variables $F(2,38)=$ $1.23, p=.30$.

\section{Discussion}

The first hypothesis was not supported. The results did not show any significant difference in the performance indicators defined in both video games. Those findings differ from those found by Cassidy and MacDonald [6]. Such results could be explained by the level of expertise of participants and the genre of games. Many studies only examined the effects of music in driving games $[6,11,13]$. The difference could be linked to the nature of the task. Playing a driving game requires skills acquired in real life, as compared to the skills required for playing a game like a FPS. Because of that, even if some participants are not considered as video game experts, their driving experience could play a role for the task.

The second hypothesis was partially supported. The item 3 "Felt happy" of the questionnaire provided significant differences between self-selected music and the other conditions, in the sense that participants reported more positive affect when exposed to self-selected music, as compared to the other music conditions. Those results are in line 
with Cassidy and MacDonald's [6] findings. However, the analysis did not confirm any significant differences for the items related to the anxiety, distraction or overall user experience, namely items 2, 6 and 7. Even though those findings are not in line with previous work $[6,11]$, it can be supposed that only the affective aspect of the enjoyment was affected by the musical preference of participants. It is plausible that the length of our games sessions, being in average two minutes each, which was relatively short, played a role in those results. It can be then assumed that duration of gameplay has an effect on attentional and cognitive factors involved in the user experience.

The third hypothesis was supported. The musical preference effect found by Cassidy and MacDonald [6] was also present in this study, in the sense that self-selected music was the most favorite for participants. This reinforce the external validity of this effect.

The fourth hypothesis was not supported. Contrary to findings of the previous literature about driving games [6], player performance was not improved by the music that would be appropriate for the game genre.

The fifth hypothesis was supported. Interactions between the type of music and the type of game presented were observed regarding music perception (items 8 and 10) and the positive affect reported by the participants (item 3 ). The low-arousal music condition was linked to better perception and higher levels of positive affect than the higharousal music condition in the puzzle game, and the opposite for the action game. One could assume that what was observed was merely an aesthetics preference regarding the arousal levels of our material. Nevertheless, self-selected music and high-arousal music were both perceived as more arousing in the action game than in the puzzle game (item 11). This provides further evidence for an interaction between the musical stimuli and the environment presented to the participant. It may mean than the perception of an auditory environment could vary in function of a person's user experience. Such results underline the importance of investigating the effects of music on video games experience, to better understand the underlying mechanisms.

\section{$5 \quad$ Conclusions and Outlook}

In this paper, the effects of self-selected music and the arousal level of music on performance and subjective experience in video games were investigated. The findings suggested that participants rate self-selected music as more appropriate, more agreeable to listen to, more arousing, and linked to more positive affect, in both the action and the puzzle games. Our results share a number of similarities with Cassidy and MacDonald's [6] findings, notably about the musical preference effect and the link between self-selected music and positive affect. Although our study did not confirm previous results on the effects of music in video game performance, it highlighted interactions between the types of music presented and the types of game in user experience.

The difference observed with the two types of games underline the importance of keeping in mind that video games do not follow a standard model when it comes to game experience, and each game will solicit different cognitive resources and trigger different behaviors depending on the players. This is part of what makes this field interesting to study, but also what raises many questions about the measurement of game 
experience $[12,14]$. The hypotheses presented to explain the difference in findings from other studies [6] should be taken into account for future studies, in order to determine the interactions between audio and game experience. Not much is known about the auditory dimension in video games [10].

With advances in technology, several video games now offer the possibility to customize the soundtrack, such as the Grand Theft Auto series (Rockstar Games) or Audiosurf 2 (Invisible Handbar, 2015). It is then worthwhile to consider that aspect of user experience during game development. Extending this practice to other types of games, such as sports games or fighting games for example, would allow both developers to manipulate the level of arousal depending of the experience intended, and players to benefit from a more tailored game experience.

\section{References}

1. Krumhansl, C.L.: Rhythm and pitch in music cognition. Psychol. Bull. 126, 159-179 (2000).

2. Cassidy, G., MacDonald, R.A.R.: The effect of background music and background noise on the task performance of introverts and extraverts. Psychol. Music. 35, 517-537 (2007).

3. Furnham, A., Allass, K.: The influence of musical distraction of varying complexity on the cognitive performance of extroverts and introverts. Eur. J. Personal. 13, 27-38 (1999).

4. Broadbent, D.: Perception and communications. Pergamon, New York (1958).

5. Nacke, L.E., Grimshaw, M.N., Lindley, C.A.: More than a feeling: Measurement of sonic user experience and psychophysiology in a first-person shooter game. Interact. Comput. 22, 336-343 (2010).

6. Cassidy, G., Macdonald, R.: The effects of music choice on task performance: A study of the impact of self-selected and experimenter-selected music on driving game performance and experience. Music. Sci. 13, 357-386 (2009).

7. Berlyne, D.E.: Aesthetics and psychobiology. Appleton-Century-Crofts, New York (1971).

8. Konečni, V.: Social interaction and musical preference. In: Deutsch, D. (ed.) The psychology of music. pp. 497-516. Academic Press, New York (1982).

9. Nantais, K.M., Schellenberg, E.G.: The Mozart effect: An artifact of preference. Psychol. Sci. 10, 370-373 (1999).

10. Caroux, L., Isbister, K., Le Bigot, L., Vibert, N.: Player-video game interaction: A systematic review of current concepts. Comput. Hum. Behav. 48, 366-381 (2015).

11. North, A.C., Hargreaves, D.J.: Music and driving game performance. Scand. J. Psychol. 40, 285-292 (1999)

12. Fang, X., Chan, S., Brzezinski, J., Nair, C.: Development of an instrument to measure enjoyment of computer game play. Int. J. Hum.-Comput. Interact. 26, 868-886 (2010).

13. Brodsky, W.: The effects of music tempo on simulated driving performance and vehicular control. Transp. Res. Part F Traffic Psychol. Behav. 4, 219-241 (2001).

14. Jennett, C., Cox, A.L., Cairns, P., Dhoparee, S., Epps, A., Tijs, T., Walton, A.: Measuring and defining the experience of immersion in games. Int. J. Hum.-Comput. Stud. 66, 641661 (2008). 